\title{
Chinese Restaurant Game
}

\author{
Chih-Yu Wang, Student Member, IEEE, Yan Chen, Member, IEEE, and K. J. Ray Liu, Fellow, IEEE
}

\begin{abstract}
In this letter, by introducing the strategic decision making into the Chinese restaurant process, we propose a new game, called Chinese Restaurant Game, as a new general framework for analyzing the individual decision problem in a network with negative network externality. Our analysis shows that a balance in utilities among the customers in the game will eventually be achieved under the strategic decision making process. The equilibrium grouping is defined to describe the predicted outcome of the proposed game, which can be found by a simple algorithm. The simulation results confirm that the rational customers in Chinese restaurant game automatically achieve a balance in loading in order to reduce the impact from the negative network externality.
\end{abstract}

Index Terms-Chinese restaurant game, game theory, Nash equilibrium, network externality.

\section{INTRODUCTION}

$\mathbf{H}$ OW rational agents make decisions in a network is an important issue in numerous research fields. One agent's decisions may be influenced by two factors: his knowledge of the system and the decisions made by other agents. The former one is determined by the agent's ability in information collecting and learning. The latter one, which is our focus in this letter, involves the network externality, i.e., the influence of other agents' behaviors on one agent's reward. Network externality is a classic topic in economics, especially in coordinate game theory [1]. The network externality can be positive or negative. When the network externality is positive, agents have higher utilities when they make the same decisions, and the problem can be modeled as a coordination game. When the externality is negative, it becomes an anti-coordination game, where agents tend to make different decisions from others for higher utilities [2]-[4].

The negative network externality plays an important rule in many applications involving resource sharing or competitions. In spectrum access problem [5], for instance, users tend to access the spectrum with less interference for better transmission quality. However, multiple agents may choose the same spectrum to access. In such a case, agents need to share the spectrum according to some access policies. Generally, the more agents access the same spectrum, the less resource each

Manuscript received May 17, 2012; revised September 24, 2012; accepted October 09, 2012. Date of publication October 18, 2012; date of current version October 25, 2012. This work was supported by the National Science Council of Taiwan under Grant NSC-100-2917-I-002-038. The associate editor coordinating the review of this manuscript and approving it for publication was Prof. Anna Scaglione.

C.-Y. Wang is with the Department of Electrical and Computer Engineering, University of Maryland, College Park, MD 20742 USA, and also with the Graduate Institute of Communication Engineering, National Taiwan University, Taipei, Taiwan (e-mail: tomkywang@gmail.com).

Y. Chen and K. J. R. Liu are with the Department of Electrical and Computer Engineering, University of Maryland, College Park, MD 20742 USA (e-mail: yan@umd.edu; kjrliu@umd.edu).

Color versions of one or more of the figures in this paper are available online at http://ieeexplore.ieee.org.

Digital Object Identifier 10.1109/LSP.2012.2225425 of them may use. This introduces the negative network externality into the problem. Another example is the deal selection problem on social networking website such as Groupon. Businesses which provide deals may receive overwhelming number of customers under the discounted deal. The overwhelming number of customers has a negative network externality on the quality of the products and therefore impairs the customers' experiences. In these examples, the negative network externality degrades the utility of the agents making the same decision. As a rational agent, one should take into account this degradation when making his decision. Note that such negative network externality exists in many different applications such as cloud computing and smart grid.

Chinese restaurant process is a non-parametric learning methods for unbounded number of objects in machine learning [6]. In Chinese restaurant process, we have a restaurant with infinite number of tables. Customers arrive the restaurant sequentially. When one customer enters, he either joins one of the tables which are already opened by other customers, or requests a new table. The probability of his decisions is related to the number of customers in each table. Generally, if a table is occupied by more customers, then a new customer is more likely to join the table, and the probability to open a new table is predetermined [7]. This process provides a systematic method to construct the parameters for modeling unknown distributions. Nevertheless, we find that Chinese restaurant process provides a proper structure to formulate the decision making problems in networks with negative externality, as previously mentioned.

By introducing the strategic behavior into the non-strategic Chinese restaurant process, we proposed Chinese Restaurant Game as a new model for social learning problems with network externality. Let us consider a Chinese restaurant with $K$ tables and $N$ rational customers. Each customer may request and be seated in any of the tables. We assume that customers prefer bigger dining space. Thus, they may prefer bigger tables. However, a customer may need to share the table with others if multiple customers request for the same table. In such a case, the customer's dining experience is impaired due to the reduced space, and the negative network externality plays a role here. Therefore, as a rational customer, one has to consider both the table size and the decisions of other customers when making his choice in tables. How rational customers make their decisions in such a game model is our main focus. In this letter, we will study the simultaneous Chinese restaurant game where customers make decisions simultaneously. The sequential Chinese restaurant game, which includes the social learning effect by letting customers make decisions sequentially, is discussed in [8].

\section{SySTEM MODEL}

We now formally formulate the Chinese Restaurant Game. Let us consider a Chinese Restaurant with $K$ tables where the size of the $i$ th table is $R_{i}$. We have $N$ customers, where each 
customer requests for one table, and they make decisions simultaneously. We first denote the table set $\mathbf{X}=\{1, \ldots, K\}$ as the action set of each customer, where $x_{i} \in \mathbf{X}$ is the choice of customer $i$. Then, the utility function of customer $i$ is given by $U\left(R_{x_{i}}, n_{x_{i}}\right)$, where $n_{x_{i}}$ is the number of customers choosing table $x_{i}$. The utility function is an increasing function of $R_{x_{i}}$ and a decreasing function of $n_{x_{i}}$. Note that the later one models the negative network externality effect since the degradation is due to the joining of other customers. Finally, let the number of customers in table $i$ be $n_{i}$, and let $\mathbf{n}=\left\{n_{1}, n_{2}, \ldots, n_{K}\right\}$ be the grouping, which plays an important role in our following analysis.

We start with a simple case where there are only two customers and two tables. The size of table 1 is $R_{1}=L$, while the size of table 2 is $R_{2}=S<L$. Given the opponent's decision, which may be the larger table or the smaller table, a rational customer should choose the table that can maximize his utility. If the decision made by the opponent is the smaller one, then the customer's best choice is the larger one since $U(L, 1)>$ $U(S, 2)$. However, if the opponent chooses the larger table, the customer's choice depends on how severe the negative network externality is. If $U(L, 2)>U(S, 1)$, the customer will choose to share the larger table. Otherwise, the smaller table will be chosen. The result shows that even both customers have perfect knowledge about the true system state, i.e., which table is larger, they may not always choose the larger one in the equilibrium. Instead, the equilibrium depends on how severe the negative network externality is. If the network externality results in an unacceptable penalty, then customers should choose different tables to avoid it.

Now let us consider the general scenario where there are $N$ customers and $K$ tables. The size of tables are given by $R_{1}, R_{2}, \ldots, R_{K}$, which are known by all customers. Since the customers are rational, their objectives in this game are to maximize their own utilities. However, since their utilities are determined by not only their own actions but also others', the customers' behaviors in the game are influenced by each other.

A strategy describes how a player will play given any possible situations in the game. In the Chinese restaurant game, the customer's strategy should be a mapping from other customers' table selections to his own selection. Recalling that $n_{j}$ is the number of customers choosing table $j$. Let us denote $\mathbf{n}_{-\mathbf{i}}=\left\{n_{-i, 1}, n_{-i, 2}, \ldots, n_{-i, K}\right\}$ with $n_{-i, j}$ being the number of customers except customer $i$ choosing table $j$. Then, a rational customer $i$ should choose the action as

$$
B E_{i}\left(\mathbf{n}_{-\mathbf{i}}\right)=\arg \max _{x \in A} U\left(R_{x}, n_{-i, x}+1\right) .
$$

The (1) describes a special set of strategy called best response, which represents the optimal action of a customer that maximizes the utility given other customers' actions. That is, given player $i$ 's action space $A_{i}$ and his utility function $u_{i}\left(x_{i}, x_{-i}\right)$, where $x_{i}$ is player $i$ 's action and $x_{-i}$ is the actions of all players except player $i$, the best response of customer $i$ is $B E_{i}\left(x_{-i}\right)=$ $\arg \max _{x \in A_{i}} u_{i}\left(x, x_{-i}\right)$.

\section{NASH EQUILIBRIUM}

Nash equilibrium is a popular concept for predicting the outcome of a game with rational customers. Informally speaking,
Nash equilibrium is an action profile, where each customer's action is the best response to other customers' actions in the profile. Since all customers use their best responses, none of them have the incentive to deviate from their actions. Formally speaking, considering a game with players $1,2, \ldots, N$. Each player $i$ has an action space $A_{i}$ and a utility function $u_{i}\left(x_{i}, x_{-i}\right)$, where $x_{i}$ is the player's action and $x_{-i}$ is the action profile of all players except player $i$. Nash equilibrium is the action profile $\mathbf{x}^{*}=\left\{x_{1}^{*}, x_{2}^{*}, \ldots, x_{N}^{*}\right\}$ where $B E_{i}\left(x_{-i}^{*}\right)=x_{i}^{*}, \forall i \in N$.

\section{A. Equilibrium Grouping}

According to the definition of Nash equilibrium, the sufficient and necessary condition of Nash equilibrium in Chinese restaurant game is stated in the following theorem.

Theorem 1: Given the customer set $\{1, \ldots, N\}$ and the table set $\{1, \ldots, K\}$, for any Nash equilibrium of the Chinese restaurant game, its equilibrium grouping $\mathbf{n}^{*}$ should satisfy

$$
U\left(R_{x}, n_{x}^{*}\right) \geq U\left(R_{y}, n_{y}^{*}+1\right), \text { if } n_{x}^{*}>0, \forall x, y \in\{1, \ldots, K\} .
$$

Proof: Sufficient condition: suppose that the action profile of all players is $\mathbf{x}=\left\{x_{1}, \ldots, x_{N}\right\}$ and such an action profile leads to the grouping $\mathbf{n}^{*}=\left\{n_{1}^{*}, \ldots, n_{K}^{*}\right\}$ that satisfies (2). Without loss of generality, let us assume that customer $i$ chooses table $j$, i.e., $x_{i}=j$, then we have $u_{i}\left(x_{i}, x_{-i}\right)=U\left(R_{j}, n_{j}^{*}\right)$. If customer $i$ chooses any other table $k \neq j$, i.e., $x_{i}^{\prime}=k \neq x_{i}=j$, then his utility becomes

$$
u_{i}^{\prime}\left(x_{i}^{\prime}, x_{-i}\right)=U\left(R_{k}, n_{k}^{*}+1\right) .
$$

Since $U\left(R_{j}, n_{j}^{*}\right) \geq U\left(R_{k}, n_{k}^{*}+1\right), \forall j, k$, we have $B E_{i}\left(x_{-i}\right)=$ $x_{i}, \forall i$. Therefore, $\mathbf{x}=\left\{x_{1}, \ldots, x_{N}\right\}$ is a Nash equilibrium.

Necessary condition: suppose that the Nash equilibrium $\mathbf{x}^{*}=\left\{x_{1}^{*}, x_{2}^{*}, \ldots, x_{N}^{*}\right\}$ leads to the grouping $\mathbf{n}^{*}=\left\{n_{1}^{*}, \ldots, n_{K}^{*}\right\}$. Without loss of generality, let us assume that customer $i$ chooses table $j$, i.e., $x_{i}^{*}=j$, therefore,

$$
u_{i}\left(x_{i}^{*}, x_{-i}^{*}\right)=U\left(R_{j}, n_{j}^{*}\right) \text { and } n_{j}^{*}>0 .
$$

If customer $i$ chooses any other table $k \neq j$, i.e., $x_{i}^{\prime}=k \neq$ $x_{i}^{*}=j$, then his utility becomes

$$
u_{i}^{\prime}\left(x_{i}^{\prime}, x_{-i}^{*}\right)=U\left(R_{k}, n_{k}^{*}+1\right) .
$$

Since $\mathbf{x}^{*}=\left\{x_{1}^{*}, x_{2}^{*}, \ldots, x_{N}^{*}\right\}$ is a Nash equilibrium, we have

$$
U\left(R_{j}, n_{j}^{*}\right) \geq U\left(R_{k}, n_{k}^{*}+1\right), \text { if } n_{j}^{*}>0, \forall j, k \in\{1, \ldots, K\}
$$

From Theorem 1, we can see that, at Nash equilibrium, one customer's utility would never become higher by deviating to another table. Moreover, any deviation to another table will degrade the utility of all customers in that table due to the negative network externality. The (2) also implies that customers may eventually have different utilities even the tables they choose have the same size. A simple example would be a three-customer restaurant with two tables in exact same size. Since there are three customers, at the Nash equilibrium, one of the table must be chosen by two customers while the other table is occupied only by one customer. 


\section{B. Uniqueness of Equilibrium Grouping}

Obviously, if there is a Nash equilibrium in Chinese restaurant game, we can always exchange the actions of any two customers in one Nash equilibrium to build a new Nash equilibrium without violating the sufficient and necessary condition shown in (2). Therefore, the Nash equilibrium is not unique. Nevertheless, the equilibrium grouping $\mathbf{n}^{*}$ can be unique as stated in the following Theorem.

Theorem 2: If the inequality in (2) strictly holds for all $x, y \in$ $\{1, \ldots, K\}$, then the equilibrium grouping $\mathbf{n}^{*}$ is unique.

Proof: We will prove by contradiction that if the inequality in (2) strictly holds, the equilibrium grouping $\mathbf{n}^{*}=\left\{n_{1}^{*}, \ldots, n_{K}^{*}\right\}$ is unique. Suppose that there exists another Nash equilibrium with equilibrium grouping $\mathbf{n}^{\prime}=\left\{n_{1}^{\prime}, \ldots, n_{K}^{\prime}\right\}$, where $n_{j}^{\prime} \neq n_{j}^{*}$ for some $j \in\{1, \ldots, K\}$. Since both $\mathbf{n}^{*}$ and $\mathbf{n}^{\prime}$ are equilibrium groupings, we have $\sum_{j=1}^{K} n_{j}^{\prime}=\sum_{j=1}^{K} n_{j}^{*}=N$. In such a case, there exists two table $x$ and $y$ with $n_{x}^{\prime}>n_{x}^{*}$ and $n_{y}^{\prime}<n_{y}^{*}$. Since $\mathbf{n}^{*}$ is an equilibrium grouping, we have

$$
U\left(R_{y}, n_{y}^{*}\right)>U\left(R_{x}, n_{x}^{*}+1\right) .
$$

Since $n_{x}^{\prime}>n_{x}^{*}, n_{y}^{\prime}<n_{y}^{*}$, and $U(\cdot)$ is a deceasing function of $n$, we have

$$
\begin{aligned}
& U\left(R_{x}, n_{x}^{*}\right)>U\left(R_{x}, n_{x}^{*}+1\right) \geq U\left(R_{x}, n_{x}^{\prime}\right), \\
& U\left(R_{y}, n_{y}^{\prime}\right)>U\left(R_{y}, n_{y}^{\prime}+1\right) \geq U\left(R_{y}, n_{y}^{*}\right) .
\end{aligned}
$$

Since $\mathbf{n}^{\prime}$ is also an equilibrium grouping, we have

$$
U\left(R_{x}, n_{x}^{\prime}\right) \geq U\left(R_{y}, n_{y}^{\prime}+1\right) .
$$

According to (7), (8), and (9) we have

$$
U\left(R_{x}, n_{x}^{*}+1\right) \geq U\left(R_{x}, n_{x}^{\prime}\right) \geq U\left(R_{y}, n_{y}^{\prime}+1\right) \geq U\left(R_{y}, n_{y}^{*}\right),
$$

which contradicts with (6). Therefore, the equilibrium grouping $\mathbf{n}^{*}$ is unique when the inequality in (2) strictly holds.

Notice that when the equilibrium grouping is not unique, there must exist some tables with equalities in (2) hold, which means that these tables become exchangeable since they offer the same expected utility in equilibrium. It will be sufficient, but not necessary to have all tables become exchangeable if all tables have the same size, which is the original assumption in Chinese restaurant process.

When the equilibrium grouping $\mathbf{n}^{*}$ is given, the utilities of customers choosing each table are also determined. Therefore, the equilibrium grouping is sufficient to describe the final state of Chinese restaurant game when we are interested in the system efficiency instead of individual utility.

\section{Algorithm FOR FINDING EQUILIBRIUM GROUPING}

We then show how the equilibrium grouping can be found through a greedy algorithm to construct one. In the original game, customers make their decisions simultaneously, which makes the outcome hard to predict. Nevertheless, the balance among tables can be easily observed by altering the game structure into a sequential game. In the proposed greedy algorithm, the customers choose their actions sequentially with customer $i$ being the $i-t h$ customer choosing the action. We let customers choose their actions in the myopic way, i.e., they choose the tables that can maximize their current utilities purely based on what they have observed. Let $\mathbf{n}_{i}=\left\{n_{i, 1}, n_{i, 2}, \ldots, n_{i, K}\right\}$ with $\sum_{j=1}^{K} n_{i, j}=i-1$ be the grouping observed by customer $i$. Then, customer $i$ will choose the myopic action given by

$$
B E_{i}^{\text {myopic }}\left(\mathbf{n}_{\mathbf{i}}\right)=\arg \max _{x \in A} U\left(R_{x}, n_{i, x}+1\right) .
$$

The proposed algorithm is shown in Algorithm 1.

\section{Algorithm 1 Finding the Equilibrium Grouping}

Input: \# of customers $N$, \# of tables $K$, table sizes $\left\{R_{k}\right\}$.

Output: Equilibrium Grouping $\mathbf{n}^{*}$ and action set $\mathbf{x}^{*}$

1: initialize $\mathbf{n}^{*}=\{0\}$

2: for $i=1$ to $N$ do

3: $U_{t}=0, x=0$;

4: $\quad$ for $k=1$ to $K$ do

5: $\quad$ if $U\left(R_{k}, n_{k}^{*}+1\right)>U_{t}$ then

6: $\quad x=k, U_{t}=U\left(R_{k}, n_{k}^{*}+1\right)$;

\section{7: $\quad$ end if}

\section{8: end for}

9: $x_{i}^{*}=x, n_{x}^{*}=n_{x}^{*}+1$;

10: end for

Theorem 3: The output grouping $\mathbf{n}^{*}$ from Algorithm 1 is an equilibrium grouping. Additionally, there exists at least one Nash equilibrium in Chinese restaurant game.

Proof: Let $\mathbf{x}^{*}=\left\{x_{1}^{*}, x_{2}^{*}, \ldots, x_{N}^{*}\right\}$ be the output action set of Algorithm 1 and $\mathbf{n}^{*}=\left\{n_{1}^{*}, n_{2}^{*}, \ldots, n_{K}^{*}\right\}$ be the corresponding grouping. For any table $j$ with $n_{j}^{*}>0$, suppose customer $k$ is the last customer choosing table $j$. Then, we have

$$
U\left(R_{j}, n_{k, j}+1\right) \geq U\left(R_{j^{\prime}}, n_{k, j^{\prime}}+1\right), \forall j^{\prime} \in\{1, \ldots, K\} .
$$

Since customer $k$ is the last customer choosing table $j$, we have $n_{j}^{*}=n_{k, j}+1$ and $n_{j^{\prime}}^{*} \geq n_{k, j^{\prime}}$. Then, according to (11), $\forall j^{\prime} \in\{1, \ldots, K\}$, we have

$$
\begin{aligned}
U\left(R_{j}, n_{j}^{*}\right) & =U\left(R_{j}, n_{k, j}+1\right) \geq U\left(R_{j^{\prime}}, n_{k, j^{\prime}}+1\right) \\
& \geq U\left(R_{j^{\prime}}, n_{j^{\prime}}^{*}+1\right)
\end{aligned}
$$

where the last inequality comes from the fact that $U(\cdot)$ is a decreasing function in terms of $n$.

Note that (12) holds for all $j, j^{\prime} \in\{1, \ldots, K\}$ with $n_{j}^{*}>0$, i.e., $U\left(R_{j}, n_{j}^{*}\right) \geq U\left(R_{j^{\prime}}, n_{j^{\prime}}^{*}+1\right), \forall j, j^{\prime} \in$ $\{1, \ldots, K\}$ with $n_{j}^{*}>0$. According to Theorem 1 and (12), the output action set $\mathbf{x}^{*}=\left\{x_{1}^{*}, x_{2}^{*}, \ldots, x_{N}^{*}\right\}$ from the proposed greedy algorithm is a Nash equilibrium. Since Algorithm 1 has only finite steps and always has outputs, the existence of Nash equilibrium is promised. Moreover, the corresponding grouping $\mathbf{n}^{*}$ is an equilibrium grouping. 


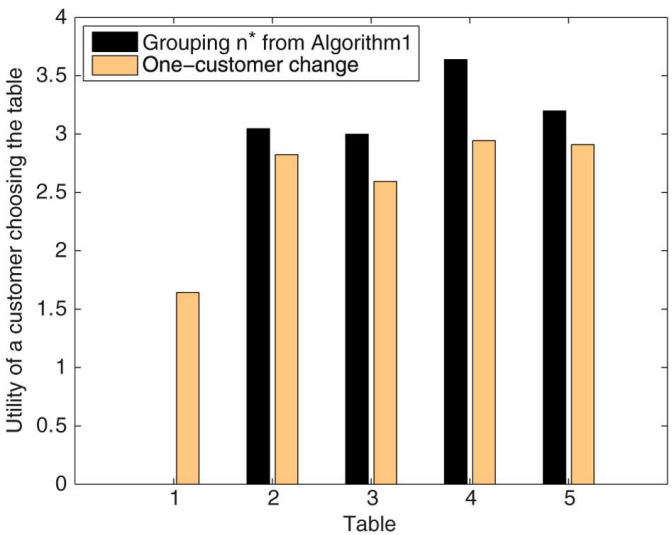

Fig. 1. Utilities under equilibrium grouping and one-customer change.

Note that the complexity of Algorithm 1 is $O(N K)$, which is quite efficient. Additionally, when the conditions in Theorem 2 hold, the output grouping $\mathbf{n}^{*}$ is the unique equilibrium grouping in the proposed game.

\section{Simulation Results}

We verify the proposed algorithm and evaluate the efficiency of equilibrium grouping in Chinese restaurant game through simulations. We consider a Chinese restaurant with 5 tables and 10 customers. The table sizes of these tables are randomly given as $[5.1636,84.1935,40.1581,38.0183,73.4684]$. The utility function is $U(R, n)=\log (R / n)$, which roughly represents the expected throughput in wireless networks if we take $R / n$ as the SINR while the interference caused by each user is 1 . We first verify if the grouping derived through the proposed algorithm is the equilibrium grouping. We compare the utility under the grouping $\mathbf{n}^{*}$ chosen by Algorithm 1 with the utilities under "one-customer change" scheme. In one-customer change scheme, the utility of customers in table $j$ is $U\left(R_{j}, n_{j}^{*}+1\right)$, which is the utility that a customer can obtain if he deviates from his original choice $x_{i}$ to table $j \neq x_{i}$. The simulation results are shown in Fig. 1. We observe that the utilities under one-customer change scheme are strictly lower than the utilities under the grouping $\mathbf{n}^{*}$. This confirms that all customers under the grouping $\mathbf{n}^{*}$ have no incentive to deviate. Therefore, the grouping $\mathbf{n}^{*}$ given by Algorithm 1 is the equilibrium grouping in Chinese restaurant game.

Next, we would like to evaluate the efficiency of the equilibrium grouping in Chinese restaurant game. We compare with two heuristic table assignment schemes: Round-Robin and Largest Table schemes. In Round-Robin scheme, customers are assigned with the table in a circular order. In Largest Table scheme, all customers choose the largest table, which is table 2 in this simulation. The utility of each customer and the averages are shown in Fig. 2. We observe that customers have balanced and generally higher utilities in Equilibrium Groping scheme, and the average utilities is highest in Equilibrium Grouping scheme among all three schemes. On the other hand, all customers in Largest Table scheme have the same utility since they all share table 2 . However, the utility is quite low due to the severe negative network externality effect under this scheme. In Round-Robin scheme, customer 1 and 6 have very low utilities because they are assigned with table 1 , which is

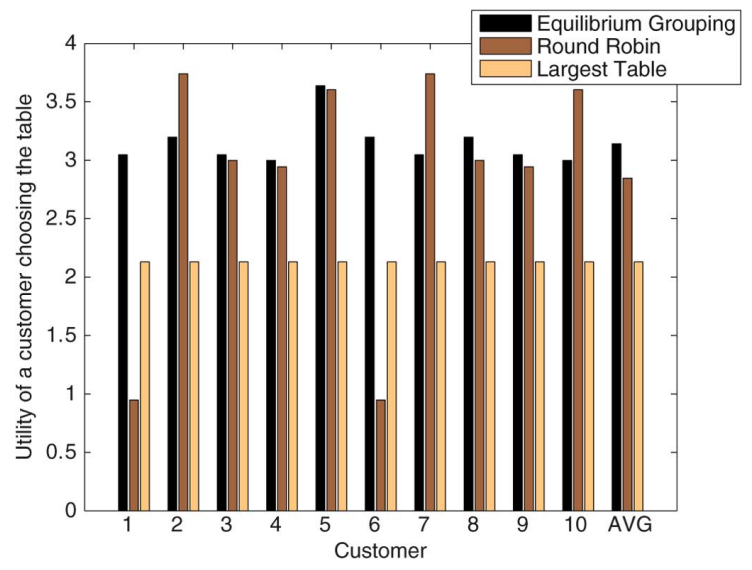

Fig. 2. Utilities of customers under different table assignment schemes

the smallest table. As a result, the average utility of customers in Round-Robin scheme is lower than the one in Equilibrium Grouping. The equilibrium grouping from Chinese restaurant game indeed balances the loading of tables, under which the customers have the highest utilities on average.

\section{CONCLUSION}

In this paper, we proposed a new game, called Chinese Restaurant Game, by introducing the strategic game-theoretic analysis into non-strategic machine learning. The proposed Chinese restaurant game can provide a new general framework for analyzing the strategic behaviors of rational agents in problems with negative network externality such as dynamic spectrum access, deal selection on Groupon and cloud service selection. By analyzing this game, we observe that the equilibrium grouping is sufficient to describe the final state of the game. The sufficient conditions for the uniqueness of the equilibrium grouping are given, and a simple algorithm to derive the equilibrium grouping is proposed in this letter. The efficiency of the equilibrium grouping from Chinese restaurant game is verified through simulations. We conclude that rational customers under Chinese restaurant game automatically achieve a balance in loadings to avoid the severe negative network externality. As a result, customers have higher utilities, and the system efficiency is enhanced.

\section{REFERENCES}

[1] R. Cooper, Coordination Games: Complementarities and Macroeconomics. Cambridge, U.K.: Cambridge Univ. Press, 1999.

[2] M. Katz and C. Shapiro, "Technology adoption in the presence of network externalities," J. Political Economy, pp. 822-841, 1986.

[3] W. Sandholm, "Negative externalities and evolutionary implementation," Rev. Econ. Stud., vol. 72, no. 3, pp. 885-915, 2005.

[4] G. Fagiolo, "Endogenous neighborhood formation in a local coordination model with negative network externalities," J. Econ. Dynam. Contr., vol. 29, no. 1-2, pp. 297-319, 2005.

[5] B. Wang, Y. Wu, and K. J. Liu, "Game theory for cognitive radio networks: An overview," Comput. Netw., vol. 54, no. 14, pp. 2537-2561, 2010 .

[6] D. Aldous, I. Ibragimov, J. Jacod, and D. Aldous, "Exchangeability and related topics," in Lecture Notes in Mathematics. Berlin/Heidelberg, Germany: Springer, 1985, vol. 1117, pp. 1-198, 10.1007/BFb0099421 [Online]. Available: http://dx.doi.org/10.1007/BFb0099421

[7] J. Pitman, "Exchangeable and partially exchangeable random partitions," Probabil. Theory Rel. Fields, vol. 102, no. 2, pp. 145-158, 1995.

[8] C.-Y. Wang, Y. Chen, and K. Liu, "Sequential chinese restaurant game," IEEE Trans. Signal Process., to be published. 